Printed in the United States of America. Avalable from National Technical Information Service

U.S. Department of Commerce

S285 Port Royal Road

Springtield, VA 2216

Price: Printed Copy $\$ 3.50$ Microfiche $\$ 3.00$

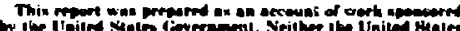

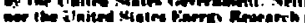

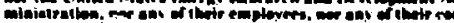

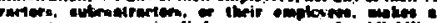

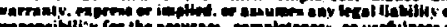

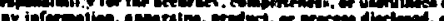

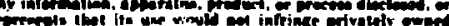

ithis. 


\section{ENGINEERING FEASIBILITY EVALUATION OF A PERISTALTIC PINCH}

by

G. P. Boicourt

PBSTRACT

A recent proposal for reducing the end loss of a linear theta panch is to produce moving magnetic mirrors at the coil ands. The concept entails the sequential pulsing of an axially arranged series of two-turn coaxial coils. The electrical design of such a systen presents some unique problems.

Ideally, the individual puise circuits should be completely independent. This would facilitate the design by eliminating interactive effects. In practice, the circuits must be interconnected through isolating inductors to enable the production of a uniform biasing magnetic field. Moreover, the coils must be located physically ciose together. This produces strong magnetic coupling between the pulse circuits, which can seriously affect the shape and speed of the inward-moving magneticmirror field.

Possible systems were modeled for the NET-2 circuit analysis code. The models took account of the inductive coupling between the individual circuits in the model.

The results show that an increasing magnetic mirror can be produced provided the radius of the theta pinch is not too great compared to the intercoil spacing. The peristaltic field can be maintained for several cycles in the inner coils. The voltage hold-off requirements on the pulse circuit switches are found to be severe, but not impossible to meet.

\section{INTRODUCTION}

Peristaltic compression has been proposed by Burke $^{1}$ to increase the compression ratio and decrease the end losses of a theta pinch. These end losses occur because the theta-pinch field is predominantly axial and the field does not act on plasma moving parallel to it. Therefore, plasma moving toward the ends is unimpeded by the field and streams out the ends of the pinch. The end losses can be reduced by placing magnetic wirrors at the ends of the theta-pinch coil. Coils positioned at the ends of a linear theta pinch are energized synchronously to produce a magnetic field that woves axially toward the center of the systen. Plasna blobs would be formed from part of the outward, axially streaning plasma and reinjected into the certtral portion of the discharge tube. The peristaltic pinch concept is not new. A similar system was discussed by Tuck $^{2}$ in 1966 . However, there appears to have been no evaluation made of this idea from the standpoint of the practical electrical circuit requirements. The following describes an evaluation of a system similar to that required by Burke.

The system evaluated was assumed constructed of presently avajlable capacitors and spark gaps. The evaluation was made by modeling the system using the NET-2 circuit analysis code. 3

\section{DESCRIPTION OF THE SYSTEM}

The syste proposed by Burke eaploys 40 twoturn coils at each end of a linear one-turn thetapinch coil. Each individual coil is of $0.64-\mathrm{cm}-\mathrm{dia}-$ weter wire and has a radius of $5 \mathrm{cn}$ with the centerto-center separation between the turns being $0.95 \mathrm{cn}$. The coils are spaced along the axis on centers 
spaced $2.54 \mathrm{~cm}$ apart. Coils at one end are sonnected in series with symetrically pos:tioned coils at the opposite end. This insures that, at the very least, these coils will operate in phase. The system chosen for analysis was the Burke system without the central theta-pinch coil. Before programming the complete system, a preliminary study was carsied olit on $1 / 8$ of the system. The $1 / 8$ system circuit is shown in Fig. 1. The combination $\mathrm{S}_{i}, \mathrm{Cl}_{1}$, and $\mathrm{il}$ represents a $10-\mathrm{kV}$ bias bank composed of $170-\mu \mathrm{F}$ capacitors. It is used to match the central coil field. The components $S 2$ and $1 . \bar{Z}$ represent a crowhar for this bank. The horizontal inductors on the left, labeled L101, L201, ..., L102, are isolating inductors used to prevent the short-circuiting of the pu'sing currents by the slow bias bank.
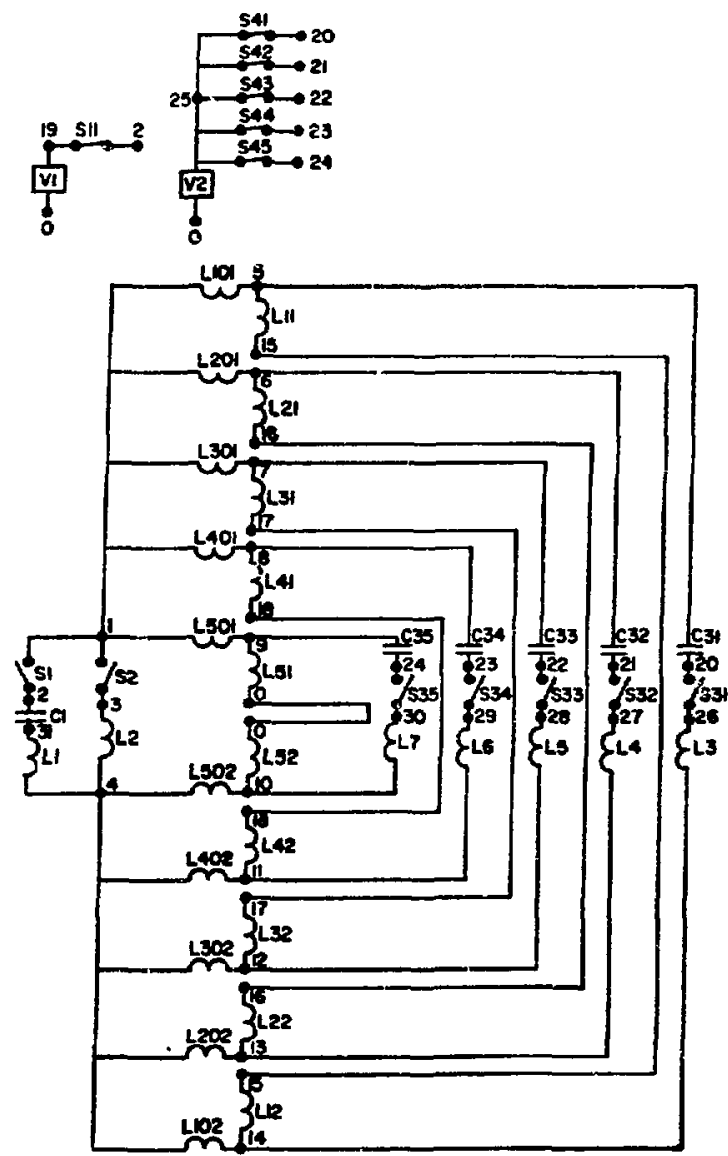

Fig. 1. One-eighth system NET-2 circuit model.
The system of 10 peristaltac coils is represented by the vertical inductors LII, L? $1, \cdots$, 112. The power source for the peristaltis coll: consists of the spark gaps, inductors, and apsit:-

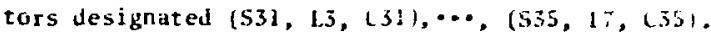
The capacitors are single, standard 1.85-jt, bu-k! units. The inductance ralues were chosen to correspond to the combined inductance of one such sapacitor in series with a standard scyluac-type sfarb. gap. The switches in these circuits are closed sequential'y to produce the wave.

The $1 / 8$ system circuit was studied $f i r$ t because positive answers from it would make further study unnecessary. Thus, if the proper traveling magnetic intensi:y can be achieved on this short system, in spite of the greater end-to-end mutual inductive coupiing, then it cas certainly be achieved on the full system. Aiso, it is much easier and cheaper to learn the idicsyncrasies of this type of circuit using a small version.

111. QULSTIONS TO BE ASSNERLD

The study was proposed to provide answers to a number of questions about the action of the circuit. The most important thing tu know about the system is whether or not it is possible for the peristaltic circuits to act independently enough to produce ahe required inward moving magnetic intensi:y wave. The close positioning of the coils results in substantial mutual inductance coupling between these circuits and this could be sufficient to prevent the formation of the wave. Given that the wave can be produced, we need to know how small the isolating inductors can be before siguificant degradation of the wave occurs and how much voltage the unfired spark gaps in the peristaltic current must hold off. This voltage will be greater than the capacitor charge voltage because of inductively induced enf's from the fired circuits. A final question is how long can the initial ph.se relation between the feristaltic circuits be maintained. For an actual plasma experiment, the initial phase relation needs to be held approximately, and for about three cycles.

\section{COMPUTATIONAL DETAILS}

In the circuit of Fig. 1, each two-turn coil is shown as a single inductor. To code the system 
for NET-2, the effective inductance of the coil and its effective mutual inductance to every other peristaltic coil in the system needs tc be calculated. The coil self-inductance ran be fould from the matrix relations

$$
\left[\begin{array}{ll}
L_{11} & L_{12} \\
L_{21} & L_{22}
\end{array}\right]\left[\begin{array}{l}
l_{1} \\
l_{2}
\end{array}\right]=\left[\begin{array}{l}
\Phi_{1} \\
\phi_{2}
\end{array}\right]
$$

This applies to the individual turns in a single coil. These are here arhitrarily denoted $I$ and 2 . The flux through the coils is, by definition, $\Phi_{\text {coil }}=L_{\text {coil }} I_{\text {coill. }}$. The coil flux is the sum of the fllix through the turn turns

$$
\phi_{\operatorname{coil}}=\phi_{1}+\phi_{2} \text {, }
$$

and the coil current is

$$
I_{\text {coil }}=I_{1}+I_{2} \text {. }
$$

Since $I_{1}=I_{2}$ because of the series connection of the turns, solution yields:

$$
L_{\text {coil }}=1 / 2\left(L_{11}+L_{12}+L_{21}+L_{22}\right) \text {. }
$$

For non-coincident coaxial coils (i.e., $i \neq j$ ), $L_{i j}$ is calculated quite accurately by the formula for circular loops,

$$
L_{i j}=\mu_{0} \sqrt{r_{i} \mathbf{r}_{j}}\{(2 / k-k) k-i / k E\} .
$$

where

$$
k^{2}=4 r_{i} r_{j} /\left\{\left(r_{i}+r_{j}\right)^{2}+\left(z_{i}-z_{j}\right)^{2}\right\} ;
$$

$K=$ complete elliptic integral of the firs $\tilde{\imath}$ kind, $E$ = complete elliptic integral of the second kind, $r_{i}=$ radius of the $i^{\text {th }}$ loop, $z_{i}=$ sxial position of $i^{\text {th }}$ loop.

For a self-inductanze of a single loop, Kirchoff's approximate formula was used,

$$
L_{i i}=\omega_{0} r_{i}\left\{\ln \frac{8 r_{i}}{a}-1.75\right\} \text {. }
$$

$a=$ the radius of the conductor.

The coefficients of coupling between the peristaltic coils can be found from

$$
m_{\alpha \beta}=M_{\alpha \beta} / \sqrt{L_{\alpha \beta} L_{\beta}} ;
$$

$M_{\alpha \beta}$ is the mutual inductance between coil $\alpha$ and coil $B$. The values of $L_{\alpha}$ and $L_{\beta}$ are found from formula (1). The mutual inductance is

$$
H_{B C:}=\epsilon_{E} / I_{a}
$$

this is the ratio of the flux in circuit $a$ due to current it, circuit a. For a system of four turns, we have th: relation

$$
\left[\begin{array}{cccc}
L_{11} & L_{j 2} & L_{13} & L_{14} \\
L_{21} & L_{22} & L_{23} & L_{24} \\
L_{31} & L_{j 2} & L_{33} & L_{34} \\
L_{4}: & J_{42} & L_{43} & L_{44}
\end{array}\right]\left[\begin{array}{l}
I_{\alpha} / 2 \\
I_{\alpha} / 2 \\
I_{B} / 2 \\
I_{g} / 2
\end{array}\right]=\left[\begin{array}{c}
\Phi_{\alpha 1} \\
\Phi_{a} \\
\Phi_{B 1} \\
\Phi_{B 2}
\end{array}\right] .
$$

Here tige first two-turn coii contains turns 1 and 2 , while the second coil contains turns 3 and 4 . Each turn carries one-half the total coil current The total flux in the second circuit, $\Phi_{B}$, is equal to the sum $\phi_{B 1}+\phi_{B 2}$. Carrying out the natrix multiplications and using the fact that we are concerned only with the flux in circuit $B$ due to $I_{\alpha}$.

$$
\phi_{B}=\phi_{B 1}+\phi_{B 2}=\left(L_{31}+L_{32}+L_{41}+L_{42}\right) I_{\alpha} / 2
$$

and $M_{B a}=1 / 2\left(L_{31}+L_{32}+L_{41}+L_{42}\right)$.

The $L_{i j}$ are computed using formula (2). The values of $m_{\alpha \beta}$ were calculated from (3) and used as input to NET-2.

The criterion chosen to evalua'e circujt performance was the magnetic field on the axis. The field was assumed to be generated by line current loops positioned at the center of each turn. Thus, each two-turn coil was treated as two loops. The axial field is given by

$$
B(z)=\frac{\mu_{0}}{2} \sum_{i=1}^{\text {No. of turns }} \frac{I_{i} r_{i}^{2}}{\left(r_{i}^{2}+\left(z-z_{i}\right)^{2}\right.} 3 / 2
$$

For a fixed $z$ position, this reduces to a weighted sun of the currents

$$
\text { 1/2 No. of coils }
$$

$$
B(2)=\sum_{\alpha=1} \quad w_{\alpha} I_{\alpha} \text {. }
$$

If the center of the coil systen is chosen as the origin, the $w_{a}$ is given by 


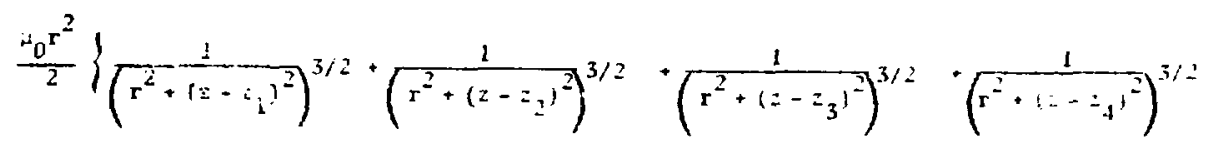

where the subsiripts 1 and 2 refer to the two turns of a single coil at one end and 3 and 4 refer to the two turns of the symmetrically positioned coil at the other end. Use of these precomputed weights reduces the work required of NET-2 and also saves space in the computer.

\section{RESULTS OBTAINED ON THE ONE-EIGHTH SYSTEM}

The initial set of runs established that the size of the isolating inductors had negligible shunting effect on the action of the peristaltic inductors provided the isolating inductors were $2 \mu \mathrm{H}$ or larger. Two microhenries is approximately s tams the tot 1 self-inductance in a single peristalta: circuit. A peristaltic field effect was also observed. This effect decreased as the firing itmes of the peristaltic circuits were brought closer together. Figure 2 gives a set of plots of the $b$ field on the axis for peristaltic circunt firngs set at $\Delta t=300 \mathrm{~ns}$ apalt. The erest of the have does not move at a speed corresponding to the firing times but the shanging slope results in an inwardly moving wave, which would push plasma toward the center. Figure 3 is a similar se: of plots for the
1/8 SYSTEM PERISTALTIC PINCH $\Delta \mathrm{t}=300 \mathrm{ne}$
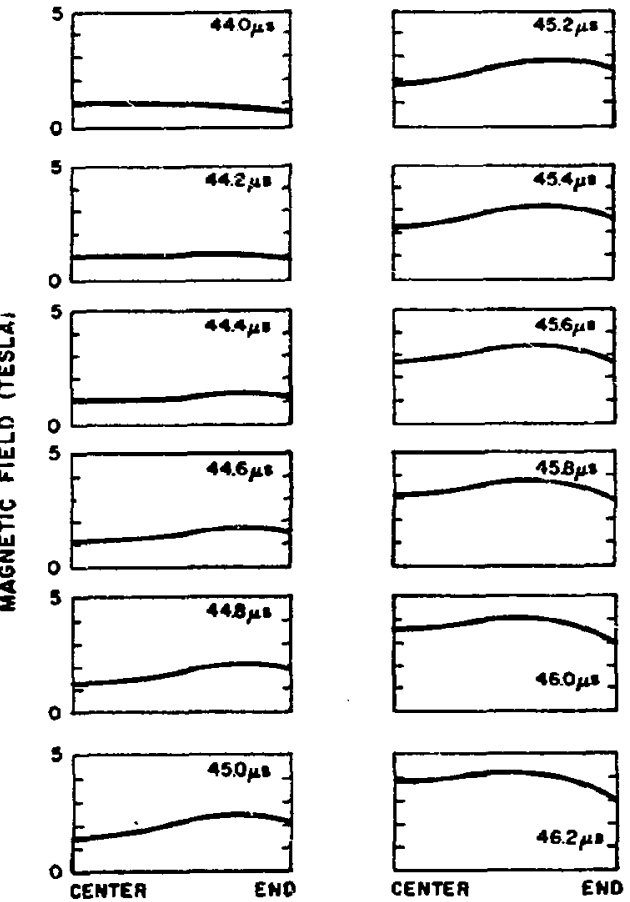

Fig. 2. Axial magnetic field intensity produced with $300 \mathrm{~ns}$ spacing between closings of the peristaltic circuit switches in the $1 / 8$ system.

\section{I/ SYSTEM PERISTALTIC PINCH $\triangle 1=100 \mathrm{~ns}$}

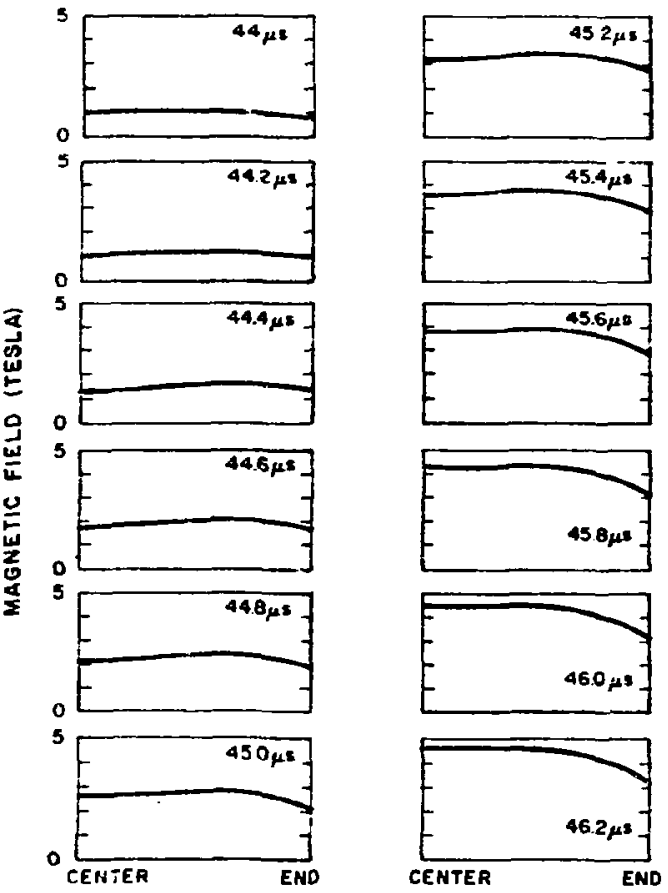

Fig. 3. Axial magnetic field intensity produced with 100 ns spacing between closings of the peristaltic circuit switches in the $1 / 8$ system. 
case where the spacing between firings is set at $\Delta t=100 \mathrm{~ns}$. Here an in-moving wave is also evident, but it is very shallow. A standard mirror coil set at the end of the theta pinch could probably do better.

It was postulated that the rapid rise of the field at the center, which results in the loss of the wave trough, was due to the strong mutual coupling between the coils. If so, this effect could be reduced by using a longer peristaltic system.

The effect of the coupling could be checked on the $1 / 8$ system by increasing the radius of the peristaltic coils. This was done by increasing the coil radii to $10 \mathrm{~cm}$. The result was that the magnetic field rose as if the coils were driven by a comon source. No peristaltic effect was noticeable. A set of curves of magnetic field intensity for this case is shown in Fig. 4.

This result shows that this type of systen depends critically on the coupling coefficients. If the coefficients are too large the machine will not work. Thus, proper operation depends critically on flux leaking out between coils. This is in contrast to a normal theta pinch where no leakage is assuned. Therefore in designing a system, care must be taken in placing other components near the peristaltic portion since, if the components are netal, they may reduce the leakage flux by blocking it.

\section{I/8 PERISTALTIC SYSTEM $\triangle 1=100_{\text {quee }}$ $20 \mathrm{~cm}$ DIAMETER COILS}

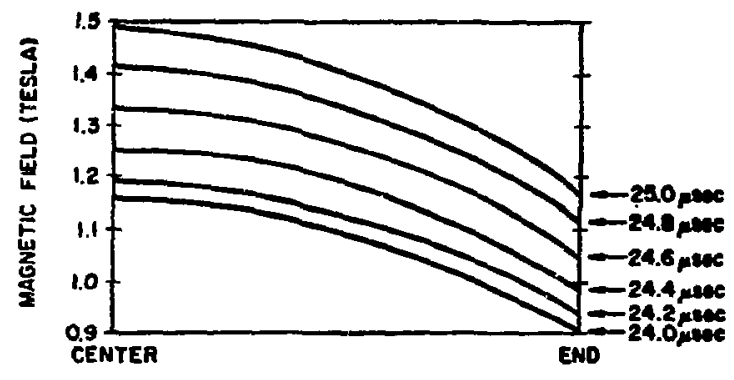

Fig. 4. Axial magnetic field intensity produced with 100 ns spacing between closings of the peristaltic circuit switches in a $1 / 8$ size system using $20-c w$-diameter peristaltic coils.

\section{LARGER SYSTEMS}

The 1/8 system model had shown that the peristaltic concept had promise but that the system wis not long enough to allow a series of inward-moving waves to be formed. To see this effect better, as well as to answer the reraining questions about the system, it was necessary to model a larger system.

A model was made of the full system. This model did not attempt to incIude all of the 3120 mutual couplings between the peristaltic inductors. Instead, only those coefficients greater than 0.00115. were retained. The attempt to solve the system failed because it was too large for the NET-2 program.

The full system was first halved and then quartered before a successful computation could be made. Some further experimentation showed that all the couplings in the 1/4 system could be included provided the anount of autput required was kept small. The 1/4 systew used all 191 mutual couplings between peristaltic jnductors--the smallest coupling coefficient was 0.000699 . The resulting NET- 2 code was large enough that the introduction of a single additional $x$ variable card resulted in a storage overflow.

A schematic of the $1 / 4$ system model is shown in Fig. S. Since the 1/8 system had settled the question concerning the size required for the

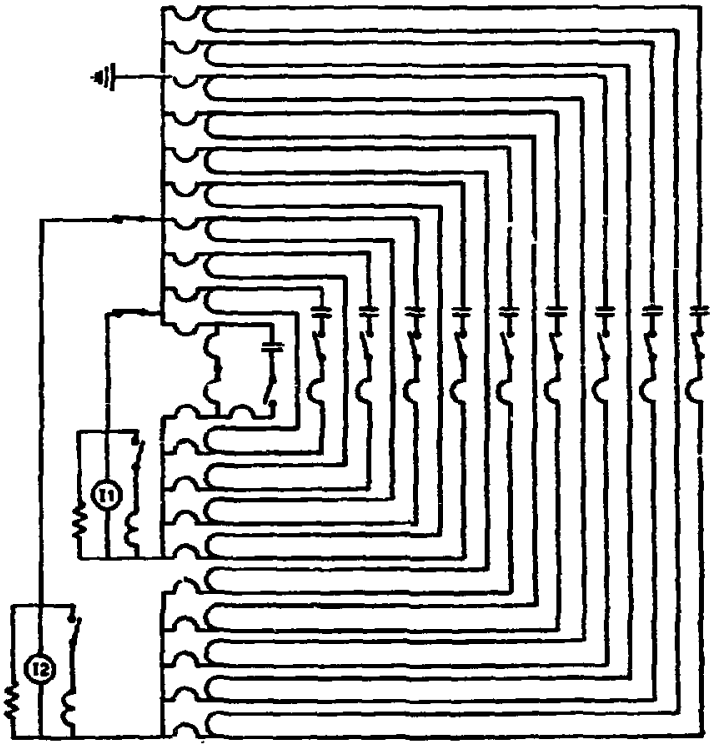

Fig. 5. One-quarter system NET-2 circuit model. 
isolating inductors, the $1 / 4$ system was modeled using current generators instead of the capacitor banks intended for use in an actual experiment. This resulted in a speed-up of the computation by eliminating the integration necessary to build up the bias current in the coils.

The central magnetic field produced in the $1 / 4$ system, when the spacing between firings $(\Delta t)$ of the peristaltic circuits was $100 \mathrm{~ns}$, 15 shown in Fig. 6 . The scale is different fron that used for the $1 / 8$ system due to the increased coil length. A comparison with Fig. 3, taking into account the scale differences, shows that the $1 / 4$ system produces a wave shape with firings spaced at $\Delta t=100 \mathrm{~ns}$, which is comparable to the shape of the $1 / 8$ system wave with $\Delta t=300 \mathrm{~ns}$. The speed of the $1 / 4$ system wave is, as expected, at least twice that of the $1 / 8$ system wave.

Figure 7 shows a longer time history of the magnetic field intensity for the $1 / 4$ system with $\Delta t$ $=200 \mathrm{~ns}$. This shows the reversal of the field and advance of the next intensity wave.

A direct extrapolation can be made to a longer system by use of this data. Such an extrapolation to a full length system is somewhat pessimistic because the longer system is less affected by the
WA SYSTEM PERISTALTIC PINCH
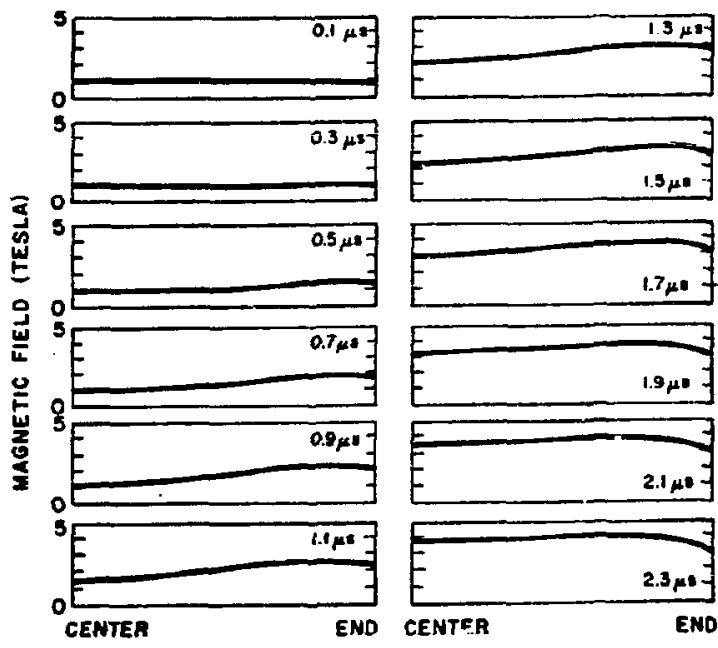

CENTER
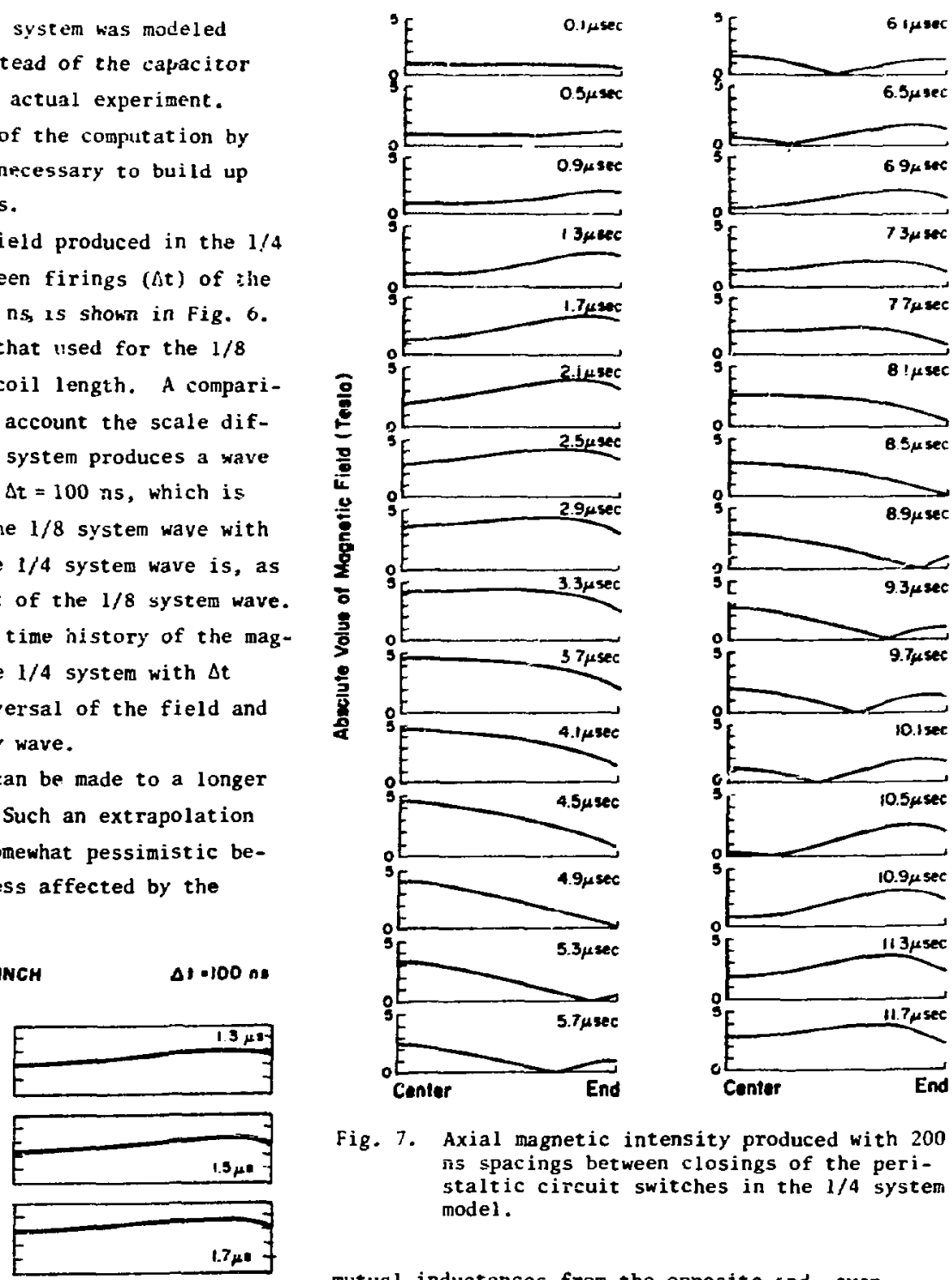

Fig. 7. Axial magnetic intensity produced with 200 ns spacings between closings of the peristaltic circuit switches in the $1 / 4$ system model.

mutual inductances from the opposite end, even though there are many more interactions. Thus, the extrapolation made from Fig. 7 and shown in Fig. 8 is worse than could be expected from the real system. It shows that in the full length system there is always an in-moving point of high field intensity somewhere along the tube.

Two questions remain to be answered. Concerning the peristaltic switches, NET-2 runs showed that the hoidmoff voltage required of the spark gaps in the peristaltic circuit was approximately $90 \mathrm{kV}$. Since 

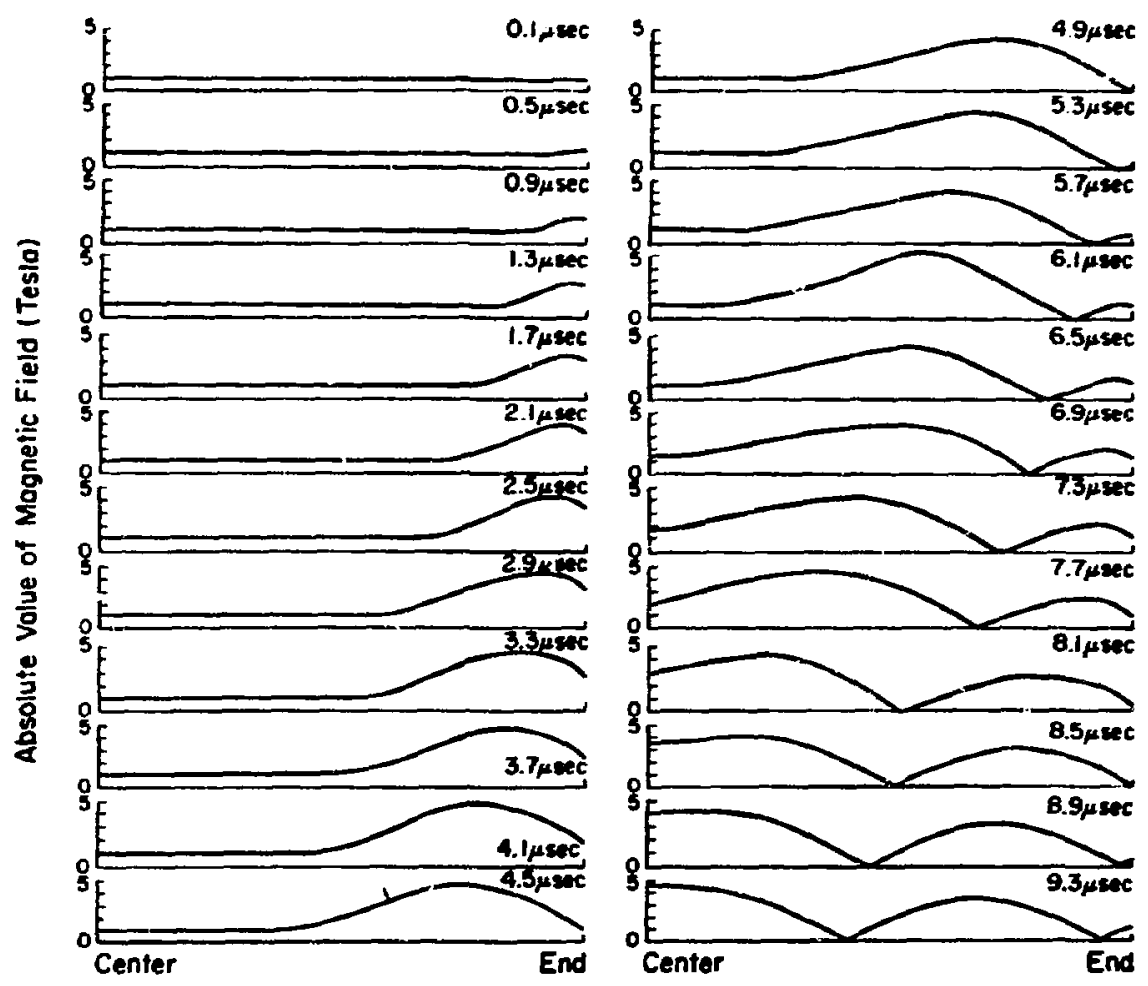

Fig. 8. Estimation of tise axial magnetic field intensity that could resul+ in a full system extrapolated from the results shown in Fig. 7.

the hold-off time is short (less than $5 \mu s$ ), this is probably within the capability of a standard Scyllactype gap.

Concerning the maintenance or the proper phase relation between the individual peristaltic circuits, long computer runs have shown that adequate independence is sustained between the circuits for more than the three cycles required for a test of the concept.

\section{SUMMARY}

This study has shown that it should be possible to construct a peristaltic pinch. The components assumed for this study are already available and hence no di jelopment should be required. The peristaltic action should be maintained for at least three cycles of peristalsis.

\section{REFERENCES}

1. R. J. Burke, "Adaptation of a Linear Theta Pinch for a FERf," Proc. of Intl. Conf. on Radiation Test Facilities for the Surface and Materials Progran, ANL, Argonne, IL, July 15-19, 1975, p. 671.

2. J. L. Tuck, "Monte Carlo and Computer Plasma Simulation Studies of the Inhibition of End loss from a Theta Pinch by Non-Adiabatic 'Rough' Magnetic Walls." Plasma Phys. and Controlled Nuclear Fusion Research, Vol. 11 (1969), p. 595.

3. A. F. Malmberg, "NET-2 Network Aralysis Program, User's Manual, Release 9," HDL-050-1, Harry Diamond Laboratories, Washington, D. C., Septenber 1973. 